\section{Zum Mechanismus der Hydroxylierung bei der Autoxidation von Zinn(II)phosphat-Lösungen}

\author{
V. Ullrich, E. Amadori * und HJ. Staudinger
}

Physiologisch-Chemisches Institut der Justus Liebig-Universität Gießen

(Z. Naturforschg. 22 b, 226 [1967] ; eingegangen am 20. Dezember 1966)

Die bei der Autoxidation vieler Komplexe niederwertiger Schwermetalle (z. B. $\mathrm{Fe}^{2 \oplus} / \mathrm{EDTA}, \mathrm{Fe}^{2 \oplus} / \mathrm{Di}$ phosphat, $\left.\mathrm{Cu}^{\oplus}, \mathrm{Ti}^{3 \oplus}, \mathrm{V}^{2 \oplus}, \mathrm{Sn}^{2 \oplus}\right)$ auftretenden Sauerstoffradikale sind in der Lage, OH-Gruppen in organische Verbindungen einzuführen. Reaktionen dieser Art wurden im Hinblick auf ihre Eignung als Modellsysteme für mischfunktionelle Oxygenasen bereits eingehend untersucht ${ }^{1,2}$.

Es ließ sich zeigen, daß die Hydroxylierung in diesen Modellsystemen über mindestens zwei Mechanismen verläuft. Einmal entstehen aus intermediär gebildetem $\mathrm{H}_{2} \mathrm{O}_{2}$ durch weitere Reduktion OH-Radikale, die Aromaten mit Substituenten 1. Ordnung elektrophil fast ausschließlich in ortho- und para-Stellung angreifen ${ }^{3}$.

Für den zweiten Mechanismus, der zu einer mehr statistischen Verteilung der Isomeren führt, wird der Angriff eines carben-analogen O-Atoms („Oxen“) diskutiert ${ }^{2,4}$. Meistens sind beide Mechanismen gleichzeitig zu beobachten.

Bei der Autoxidation von Sn(II) -phosphat-Lösungen scheint die Hydroxylierung jedoch nur nach dem „Oxen-Mechanismus“" zu erfolgen, da die aus Acetanilid, Anisol, Phenetol, Toluol und Phenacetin entstehenden Phenole die für diesen Mechanismus charakteristische, rein statistische Verteilung zeigen (Tab. 1). Auch Aromaten mit Substituenten 2. Ordnung, die mit $\mathrm{OH}-$ Radikalen schlechter reagieren, werden in gleicher Weise umgesetzt (Tab. 2). Weiterhin findet man beim 2-Methylbutan, dessen primäre und sekundäre CH-Bindungen von $\mathrm{OH}$-Radikalen nicht angegriffen werden, mit diesem System eine Verteilung der $\mathrm{C}_{5}$-Alkohole, wie sie für die Einschiebung von O-Atomen in CH-Bindungen zu erwarten ist (Tab.3). Die Sonderstellung des Systems $\mathrm{Sn}^{2 \oplus} / \mathrm{HPO}_{4}{ }^{2}{ }^{\ominus} / \mathrm{O}_{2}$ erklärt sich daraus, daß $\mathrm{H}_{2} \mathrm{O}_{2}$ als eines der Zwischenprodukte der Sauerstoffreduktion nicht wie in den übrigen Systemen zu $\mathrm{OH}$ Radikalen, sondern sofort zu $\mathrm{H}_{2} \mathrm{O}$ reduziert wird. Einzelheiten dieser Ergebnisse werden an anderer Stelle veröffentlicht.

* Aus der Dissertationsarbeit von E. Amadori.

1 S. Udenfriend, C. T. Clark, J. Axelrod u. B. B. Brodie, J. biol. Chemistry 208, 731 [1954].

2 Hu. Staudinger u. V. Ullrich, Angew. Chem. 77, 924 [1965].

\begin{tabular}{|c|c|c|c|c|}
\hline System & $\begin{array}{c}\text { Hydro- } \\
\text { xylie- } \\
\text { rungs- } \\
\text { produkte } \\
{[\%]}\end{array}$ & $\begin{array}{l}\text { Acet- } \\
\text { anilid }\end{array}$ & Anisol & Toluol \\
\hline 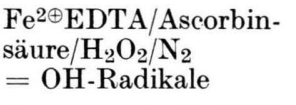 & $\begin{array}{l}\text { ortho } \\
\text { meta } \\
\text { para }\end{array}$ & $\begin{array}{c}46,5^{5} \\
2,5 \\
51,0\end{array}$ & $\begin{array}{r}66 \\
0 \\
34\end{array}$ & $\begin{array}{l}59 \\
10 \\
31\end{array}$ \\
\hline $\begin{array}{l}\mathrm{Sn}^{2 \oplus} / \mathrm{HPO}_{4}^{2} \ominus / \mathrm{O}_{2} \\
=,, \mathrm{Oxen} * \\
{\left[0, \mathrm{OH}^{\ominus}\right]}\end{array}$ & $\begin{array}{l}\text { ortho } \\
\text { meta } \\
\text { para }\end{array}$ & $\begin{array}{l}34 \\
44 \\
22\end{array}$ & $\begin{array}{l}36 \\
47 \\
17\end{array}$ & $\begin{array}{l}46 \\
37 \\
17\end{array}$ \\
\hline
\end{tabular}

Tab. 1. Bestimmung der Isomerenverteilung. Acetanilid: HJ. Staudinger u. V. Ullerich, Z. Naturforschg. 19 b, 409 [1964]. Anisol: gaschromatographisch mit Perkin Elmer F 6, Säule: 5\% Äthylenglycolsuccinat auf Chromosorb 60/80 mesh, $200 \mathrm{~cm}$; Trägergas $\mathrm{N}_{2}, 170^{\circ} \mathrm{C}$, FID (E. Amadori, Diss., in Vorbereitung). Toluol: gaschromatographisch mit Perkin Elmer F 6, abgewandeltes Verfahren (Trägergas $\mathrm{N}_{2}$, FID) von W. Sassenberg u. K. Wrabetz, Z. analyt. Chem. 184, 423 [1961].

\begin{tabular}{|c|c|c|}
\hline System & $\begin{array}{l}\text { hydroxylie- } \\
\text { rendes } \\
\text { Teilchen }\end{array}$ & $\begin{array}{c}\text { Relative } \\
\text { Reaktivi- } \\
\text { täten } \\
\text { Acetanilid/ } \\
\text { Benzoesäure }\end{array}$ \\
\hline 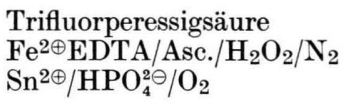 & $\begin{array}{c}{\left[\mathrm{OH}^{\oplus}\right]} \\
\left.\cdot \mathrm{OH}^{-\mathrm{OH}^{\ominus}}\right]\end{array}$ & $\begin{array}{r}55 \\
3,5 \\
1,2\end{array}$ \\
\hline
\end{tabular}

Tab. 2. Bestimmung der Hydroxybenzoesäuren: dünnschichtchromatographisch (V. Ullrich, unveröff. Versuche). Asc. = Ascorbinsäure.

\begin{tabular}{|c|c|c|c|c|}
\hline \multirow[t]{2}{*}{ System } & \multirow[t]{2}{*}{$\begin{array}{l}\text { hydroxylie- } \\
\text { rendes } \\
\text { Teilchen }\end{array}$} & \multicolumn{3}{|c|}{$\begin{array}{c}\text { Reaktivitäten } \\
\text { gegen } \\
\text { 2-Methylbutan }\end{array}$} \\
\hline & & $\mathrm{CH}$ & $\mathrm{CH}$ & $\mathrm{CH}$ \\
\hline \multirow{3}{*}{ 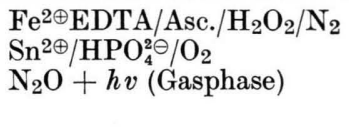 } & $\cdot \mathrm{OH}$ & - & - & 1,0 \\
\hline & {$\left[\mathrm{O}, \mathrm{OH}^{\ominus}\right]$} & 1,2 & 1,0 & 2,8 \\
\hline & $\begin{array}{l}\mathrm{O}\left({ }^{1} \mathrm{~S}\right) \\
\mathrm{O}\left({ }^{1} \mathrm{D}\right)\end{array}$ & 1,1 & 1,0 & 1,0 \\
\hline
\end{tabular}

Tab. 3. Bestimmung der Isopentanole: gaschromatographisch, Verfahren von B. Drews, H. Specht u. G. OfFer, Z. analyt. Chem. 189, 325 [1962]. Asc. $=$ Ascorbinsäure.

${ }^{3}$ R. O. C. Norman u. J. R. Lindsay Smith, in: Oxidases and Related Redox Systems. Wiley, New York 1965, Bd. I, S. $131 \mathrm{ff}$.

${ }^{4}$ G. A. Hamilton, J. Amer. chem. Soc. 86, 3391 [1964].

5 H.. Staudinger u. V. Ullaich, Z. Naturforschg. 19 b, 409 [1964]. 